\title{
La historia de la forma de la revista a través del prisma de la historia de la "gran conversación científica"*
}

Entrevista con Jean-Claude Guédon dirigida por Alain Loute y preparada con la ayuda de Caroline Glorie, Thomas Franck y Andrea Cavazzini

\section{Del comercio epistolar a las publicaciones de las academias}

Jean-Claude Guédon: El término revista científica, en realidad, cubre objetos muy diferentes en la historia. En mi opinión, y como lo menciono en mi texto Open Access: Toward the Internet of the Mind, ${ }^{1}$ la revista científica moderna que aparece en el siglo XVII corresponde a una mecanización del régimen de escritura de cartas que caracterizó buena parte de los intercambios científicos de finales del Renacimiento y comienzos de la llamada era "moderna". La imprenta creó un sistema de comunicación más eficiente que comenzó a desmoronarse bajo el peso de los intercambios postales. Siempre olvidamos esta importante revolución en la Europa del Renacimiento, que es la invención del correo. A partir de éste, se permite que toda una categoría de personas alfabetizadas comience un intercambio de información. Ahora los límites demográficos de las ciudades son superados, el mercado de ideas puede expandirse considerablemente y la "gran conversación científica" puede intensificarse.

Por lo tanto, la revista científica impresa aparece primero como una forma mecanizada del correo. Al mismo tiempo, se vuelve dependiente y víctima, diría yo, de la forma que la imprenta impone al documento. Una cosa que siempre pienso importante de señalar es que cuando aparece la imprenta, lo hace a la par la mercantilización del documento. No se puede imprimir sin dar

* Originalmente publicada en la revista Les Cahiers du GRM, a quien agradecemos la autorización de esta traducción. DOI: https://doi.org/10.4000/grm.912 
a los documentos un formato comercializable simplemente porque se tiene que invertir previamente en equipos pesados y porque se emplea una gran variedad de especialistas. Simplemente pasee por el museo imprenta Plantin-Moretus en Amberes (Bélgica); después de ello, aparece muy claramente una forma de integración vertical que parte de los metalúrgicos que preparan las fuentes para los letrados encargados de revisar sistemáticamente los textos, a veces en cuatro, cinco o seis idiomas. Imprimir un texto requiere de un enredo extremo entre el mundo financiero y el mundo de la producción.

Las primeras revistas científicas se encuentran en este sistema. Para el ejemplo francés del Journal des sçavans, la dimensión comercial de la empresa es bastante evidente. En Inglaterra, la forma de la primera revista científica, The Philosophical Transactions of the Royal Society, se basa en un modelo mixto y más complicado que revela otras dimensiones emergentes de las revistas científicas. En particular, su filiación a una academia real la ubica de una manera original, incluso si la sociedad real de Londres no se hizo cargo inmediatamente de ella. Fue hasta a mediados del siglo XVIII cuando, después de apoyarla y refrendarla, tomó responsabilidad financiera de ella. Esto último puede verse como una demostración del hecho de que, incluso si tiende a imponer un esquema comercial en la comunicación científica, la imprenta requiere de algo más, por lo que puede volverse autónoma en relación con los objetivos comerciales.

En Francia, la Real Academia de Ciencias tendrá su propia prensa y sus propios privilegios, lo cual es bastante extraordinario. El poder real podía contar con el hecho de que los filósofos de la naturaleza hablarían de la naturaleza, sin gran riesgo de subversión política. De ahí que se dejara bastante tranquilos a estos filósofos de la naturaleza que generalmente respetaron las reglas. Debe recordarse que, en Inglaterra, la Royal Society nació después del paréntesis parlamentario de Cromwell, y mantenerse 
alejada de las cuestiones políticas fue simplemente la más elemental de las sabidurías. Por otro lado, el conocimiento científico no siempre ha podido evitar un diálogo difícil con la religión. Mi colega Yves Gingras, de la Universidad de Quebec, en Montreal, acaba de publicar un excelente libro sobre este tema. ${ }^{2}$

Resumamos este primer logro: la revista científica comienza como un dispositivo epistolar mecanizado y mercantilizado. Al mismo tiempo, la revista científica está tratando de empoderarse en relación con la esfera comercial, especialmente cuando es apoyada por academias. La situación se vuelve más ambigua en el caso de las pequeñas empresas privadas. Los textos más reconocidos en los siglos XVII y XVIII aparecieron en las academias o gracias a ellas. La Real Academia de Ciencias de París fue refundada en 1699 y, a partir de ese momento, comenzó a publicar sus imponentes volúmenes de historias y memorias.

Las raíces académicas de las revistas académicas explican cómo se ven posicionadas en la trayectoria general del esfuerzo de investigar. La revista científica no constituye una fase separada en esta trayectoria; por el contrario, constituye una parte esencial. Tuve algunos debates con colegas estadounidenses sobre este punto porque querían absolutamente tratar la fase de publicación científica como si fuera una fase de investigación completamente diferente del resto, lo que justificaría la forma comercial de estas revistas. Sin embargo, la investigación sin publicación no tiene sentido, y la publicación sin investigación, aún menos. Con ayuda de las academias que aumentan en número en la Europa de la llustración, se multiplican las revistas que dominan la producción científica de esta parte del mundo hasta al menos alrededor de 18201825.

\section{El apogeo de las sociedades académicas y sus revistas científicas}


Entre 1825 y 1945 o 1950, la historia de las revistas científicas estuvo regida por un nuevo régimen de publicación particularmente interesante hoy en día. El científico como un rol social particular emerge y se define en varias sociedades europeas de este tiempo; en Inglaterra, esta transformación está marcada por la aparición de la palabra scientists. También fue el momento en que los scientists aparecieron en todas partes y, como resultado, se encontraron algo aislados en las provincias y en los pueblos pequeños. Luego buscan aumentar el alcance y la importancia de su trabajo y, para hacerlo, se organizan en asociaciones científicas. Posteriormente se crea un movimiento notable y difundido en Europa, aunque particularmente en Inglaterra. En este último país, estas asociaciones se unen a nivel nacional bajo el paraguas general de la British Association for the Advancement of Science (BAAS). Más tarde, Francia seguirá con l'Association Française pour l'Avancement des Sciences (AFAS). BAAS y sus imitadores constituyen un movimiento que, de manera muy inteligente, 1) reconoce la existencia de comunidades científicas, 2) reconoce la importancia de la comunicación entre los científicos, 3) reconoce que la dispersión de los científicos dificulta el punto 2 y que 4) se deben encontrar formas de superar estos obstáculos. Hay dos formas para alcanzar esto y de las cuales los británicos también son pioneros. Primero, un congreso itinerante cubre primero Gran Bretaña y eventualmente se extiende a toda la Commonwealth. Se desarrollan estrategias similares en los otros países de Europa. En segundo lugar, los miembros de estas asociaciones contribuirán en el financiamiento de la publicación de una revista académica y seleccionarán sus mejores trabajos para publicitarlos en el extranjero. Para proyectarlos en el exterior, se utiliza la forma aparentemente comercial de la suscripción, aunque, por supuesto, esta también es acompañada de un intercambio de revistas entre asociaciones. Una revista con un tiraje de 500 ejemplares permite recoger varios cientos de revistas pertenecientes a otras asociaciones. Muy rápidamente, a través de este medio tan simple, uno puede hacerse de una buena biblioteca básica y seguir así algunos de los mejores trabajos científicos en Europa en las principales lenguas del continente. No obstante, dicho sistema también requiere herramientas de seguimiento; para ello, se desarrollan resúmenes y bibliografías. A diferencia de las publicaciones académicas, estas herramientas 
tienden a ser completamente comerciales. También se observan esfuerzos en la traducción, especialmente en los países cuyos idiomas son poco conocidos o no se usan ampliamente. En esta nueva configuración de comunicación científica, ciertos idiomas comienzan a ocupar un lugar importante: el alemán, el inglés y el francés ciertamente forman parte de este grupo. El ruso y el italiano tampoco están ausentes.

Al mismo tiempo, se construye una creciente conciencia del científico que ahora es miembro de una comunidad internacional. Las cuestiones de la universalización del conocimiento científico adquieren entonces un nuevo sentido; yendo más allá del nivel epistemológico, la internacionalización de la ciencia adquiere un significado más bien sociológico e institucional. La ciencia se puede hacer en cualquier lugar, por cualquier persona y siempre y cuando se respete un cierto número de cosas. Estamos comenzando a ver la aparición de formas de comportamiento que Merton, en la época de la Segunda Guerra Mundial, abordará en sus primeros trabajos de sociología de la ciencia.

Las revistas de asociaciones científicas también adolecen de deficiencias, por ejemplo, en los métodos de gestión utilizados y en los problemas encontrados que les abrirán oportunidades en el sector comercial. Dos elementos comienzan a jugar, especialmente en el caso de las academias, pero también dentro de ciertas asociaciones: básicamente estas no son editoriales y no siempre poseen las técnicas necesarias, lo que puede afectar, por ejemplo, la velocidad de sus publicaciones. Algunas de estas revistas, aunque prestigiosas e importantes, comienzan a rezagarse. Simplemente no van lo suficientemente rápido como para satisfacer las necesidades de la investigación científica y, sobre todo, para hacer frente a aquellas creadas por la creciente competencia entre científicos.

Más allá de los problemas de eficiencia en el orden de la producción que encuentran las revistas de las sociedades científicas, la cuestión de su relación con la política sigue siendo todo un tema. La estadística, por ejemplo, puede conducir a la revelación del terrible estado en el que se encuentran los trabajadores ingleses, y esto, evidentemente, no con el fin de complacer al poder real. También hay formas 
de conocimiento en disputa, como la frenología, que deben ser abordadas con reserva. Por lo tanto, por diversas razones, las revistas académicas vinculadas a sociedades se posicionan con dificultad frente a diversas formas del conocimiento. Este tipo de decisión, de hecho, requiere de la creación de límites y demarcaciones que no siempre serán fáciles de legitimar o justificar. Destacarse entre los ámbitos científico y no científico también puede equivaler, en ciertas circunstancias, a efectos de censura.

Con la falta de velocidad, una posible censura y otros aspectos más, los vendedores se dieron cuenta de lo potencialmente rentable que podría ser la publicación de obras que estaban limitadas a lectores de temas esotéricos y que, por consiguiente, eran difíciles de comprender por los no especialistas. Desarrollé un poco este punto en mi antiguo texto de 2001, "Dans l'ombre portée de Oldenburg". ${ }^{3}$ Oldenburg, recordemos, era el secretario de la revista Philosophical Transactions en Londres. Como explico en el texto, en el siglo XIX estas editoriales, en particular holandesas, alemanas $y$, en cierta medida, inglesas y francesas, apoyaban o desarrollaban revistas, no tanto para ganar dinero, sino sobre todo para mantener vivo un vínculo con posibles autores de los cuales podrían esperar, de alguna manera, obtener frutos. En efecto, el objetivo era elegir mejor y con mayor eficacia a los autores que podrían escribir libros, ya que las monografías científicas demoraban la forma de búsqueda preferida de ganancias en las publicaciones científicas del siglo XIX. Además, los científicos aún publicaban muchos libros en el siglo XIX y principios del siglo $\mathrm{XX}$; pensemos en Darwin, por ejemplo. $\mathrm{Y}$ no estoy hablando de las humanidades y de las letras aquí. En las ciencias naturales, el encanto por las monografías se mantuvo fuerte, situación que continúa hoy en las matemáticas.

En estos esfuerzos por comercializar publicaciones científicas, Alemania desempeñará un papel de liderazgo durante fines del siglo XIX y principios del XX. La Primera Guerra Mundial y sus consecuencias frenarán la publicación de ciencia en lengua alemana, pero es sobre todo con el nazismo como dará comienzo una transición en forma de emigración continua no sólo de científicos alemanes de religión judía, sino también de editoriales científicas en manos de familias judías. 
Esto nos lleva al borde de una transición importante en el orden de las publicaciones científicas.

\section{III. ¿Cómo han logrado las revistas especializadas ocupar una posición dominante en las publicaciones científicas?}

En la historia de las revistas científicas, una tercera fase comienza al final de la Segunda Guerra Mundial. La investigación científica fue absolutamente fundamental para el resultado de la guerra. El ejemplo más llamativo es la bomba atómica. Conocemos todos los esfuerzos realizados por los científicos de ambos lados para tratar de encontrar armas "decisivas". Al final de la guerra, los alemanes, por su parte, habían inventado el avión de reacción y los cohetes balísticos. En resumen, ya estábamos entrando al siglo XXI entre 1944 y 1945. Con la Guerra Fría, las inversiones en investigación científica explotaron, tanto entre los soviéticos como entre los estadounidenses, y luego, cuando comenzó a recuperar el aliento, también en Europa. Esto condujo a una demanda de publicaciones a la que las sociedades y academias no pudieron responder. Éste fue el trasfondo que permitió a los editores comerciales ingresar al mundo de las publicaciones académicas.

A pesar de esto, la explosión de la investigación científica no es la única explicación para la entrada de vendedores en el mundo de la investigación. Un segundo elemento se encuentra en el hecho de que la investigación científica, después de la Segunda Guerra Mundial, tomó una forma mucho más multidisciplinaria o interdisciplinaria. En resumen, la solución de problemas complejos tenía prioridad sobre el desarrollo ordenado de campos disciplinarios. Como resultado, las herramientas convencionales para localizar información científica rápidamente demostraron ser insuficientes, particularmente en campos emergentes, como la genética o la biología molecular. Las bibliografías en química o biología ya no respondían a las preguntas de los investigadores. Es decir, ya no se sabía cómo seguir de cerca los desarrollos que involucraban varias disciplinas. 
Aquí es donde se configura un sistema de seguimiento de información completamente nuevo. Éste fue inventado por Eugene Garfield bajo el nombre de Science Citation Index. Este sistema permitió a los investigadores encontrar información rastreando citas antes que cualquier publicación académica. Este sistema de seguimiento ignoró los límites disciplinarios para seguir sólo los rastros de información realmente utilizada en un problema de investigación en particular.

Para rastrear las citas de artículos científicos y luego clasificarlas en las computadoras de los años 1960, Garfield tuvo que realizar un truncamiento severo de las publicaciones científicas mundiales, para llegar así a un inventario riguroso de citas de un número reducido de publicaciones. A pesar de esto, tuvo que ser capaz de demostrar que este truncamiento permitía identificar anteriores e importantes trabajos sin pérdidas significativas. Por lo tanto, el número limitado de publicaciones que Garfield y su equipo rastreaban en realidad tenía que parecer un reflejo confiable, y sobre todo creíble, de toda la publicación científica mundial. Garfield superó este desafío vital para el futuro de su negocio inventando, lo que de hecho es sólo un mito: las revistas centrales de ciencia. En su opinión, estas revistas representaban la esencia de la ciencia y eran suficientes para estudiar trabajos previos. ¿Cómo funcionan en realidad? Éstas se basan en una antigua ley de bibliografías que fue inventada en 1934, en Gran Bretaña, por un hombre llamado Samuel C. Bradford. De hecho, esta ley tenía como objetivo el retorno de los esfuerzos de un investigador para encontrar artículos relevantes para una investigación dada en la literatura en general. Bradford explica que, si se buscan artículos sobre un tema determinado, se encontrará rápidamente un cierto número de artículos en un número reducido de revistas; si luego queremos duplicar el número de artículos encontrados, tendremos que examinar un número de comentarios igual al cuadrado del número inicial de comentarios, y así sucesivamente. En consecuencia, el intento de aumentar aritméticamente el número de artículos relacionados con un tema va acompañado de la necesidad de consultar una serie de revistas que, a su vez, aumenta geométricamente. 
La ley de Garfield Bradford lo revertirá de alguna manera. Imagine, nos dice, una reunión de bibliotecarios. Todos llegan con la lista de revistas favoritas correspondiente a las necesidades de su institución. Están sentados alrededor de una mesa, en una habitación donde las ventanas están abiertas. Es el verano. De repente, hay una tormenta eléctrica y una gran ráfaga de viento barre todas las listas haciendo que éstas vuelen y se mezclen. Una vez que la tormenta ha pasado, los bibliotecarios, lo mejor que pueden, recogen las listas dispersas y es entonces cuando todos se dan cuenta de que cada uno tenía esencialmente la misma lista. La ley de Bradford, que es una ley de rendimientos decrecientes en relación con la investigación, según Garfield, puede revertirse, ya que de hecho se basa en la tesis implícita de que todos tienen aproximadamente la misma visión sobre qué revistas son importantes en la ciencia.

Armado con su fábula, Garfield de hecho introduce la idea de que el mundo entero, cualquiera que sea el país, obedece a una especie de consenso tácito sobre todas las preguntas que importan en un momento dado de la historia de la ciencia. Sin embargo, esta tesis introduce una noción de universalismo diferente de la que Robert Merton plantea en su caracterización de los valores fundamentales de la práctica científica. Mientras que Merton definió el universalismo como la posibilidad de que cualquier individuo participe en el esfuerzo científico, sin especificar la naturaleza o dirección de este esfuerzo, Garfield reconstruye el universalismo en forma de una especie de convergencia tácita universal entre científicos, donde todas las preguntas importantes en la ciencia se expresarían en un momento de su historia. Sin embargo, en cualquier momento de su historia las ciencias pueden plantear muchos más problemas de los que los científicos pueden resolver. Además, la idea misma de una política científica nacional contradice al menos parcialmente esta tesis. En una última instancia, esto equivaldría a decir que las revistas científicas, con sus diversas orientaciones editoriales, solo reflejarían esta extraña visión del universalismo. En otras palabras, esta visión es extraña porque la ciencia, en lugar de estar abierta a cualquier ser humano que acepte seguir el método científico como lo concibió Merton, es en realidad un conjunto de problemas que competen a un grupo privilegiado e influyente de investigadores, directores de 
grandes laboratorios y editores en jefe de revistas científicas altamente visibles. Es en esta nueva perspectiva sobre el "universalismo" en la que en todos lados se necesitará el mismo corpus documental. Este último punto es introducido sotto voce por Garfield. De hecho, éste afirma haber encuestado a sus colegas (la gran mayoría de los cuales eran estadounidenses), a los cuales, a su vez, pidió que hicieran lo mismo con otros colegas, y así sucesivamente, acordando que se hicieran siempre las mismas preguntas en genética, física, química, etc. Una vez que la literatura científica se hubiera homogeneizado de esta manera, sólo quedaba eliminar la mayoría de las revistas del mundo mediante la identificación de las más importantes, aquellas de las cuales habría de emplear el vocabulario.

¿Es útil resaltar los problemas asociados con este enfoque? Los investigadores estadounidenses y europeos entrevistados trabajaron en países en situaciones de desarrollo social más o menos equivalentes, con los mismos tipos de preguntas por resolver. Por ejemplo, es probable que si, en el momento de la constitución del Science Citation Index, Garfield hubiera interrogado a los investigadores sobre la cuestión del cólera, probablemente habrían respondido que este problema ya había sido resuelto y que ya no era necesario hablar de él. Sin embargo, en muchas partes del mundo les habría gustado hablar de ello. Y si se hubiera deseado trabajar en este problema, habría sido difícil encontrar un periódico central que aceptara dicho tema. ¿Qué interés tendría para la mayoría de sus lectores? Sin embargo, la idea de Garfield sostenía que la ciencia tendría un conjunto universal de preguntas o problemas adecuadamente abordados por el nuevo género del universalismo científico.

Esta forma de hacer las cosas, de hecho, deja muchas preguntas sin responder, preguntas simplemente pasadas por alto, olvidadas. Por ejemplo, el virus zika fue aislado en 1947 y el del ébola se identificó en 1976. Sin embargo, estas enfermedades se han descuidado durante décadas. ¿Por qué han sido pasadas por alto? Por un lado, las personas que trabajan en los centros dominantes de la ciencia no abordan espontáneamente estas preguntas, ya que las preguntas más "rentables" en términos de subvenciones y promociones son aquellas que 
probablemente atraerán una alta visibilidad y que se sitúan en otros ámbitos. Al mismo tiempo, si los investigadores en países donde abundan los virus como el ébola y el zika, es decir, países pobres, llegan con dificultad y miseria a ofrecer preguntas y algunas respuestas sobre estas enfermedades, tendrán dificultades para publicar en otros sitios que no sean pequeñas publicaciones locales. Y, por otro lado, en los países en donde la ciencia "cuenta", estos resultados pasarán desapercibidos.

Por lo tanto, el resultado del sistema que Garfield ayudó a establecer comienza a instaurar, o más bien a endurecer, una situación en la que, de hecho, se distinguen dos tipos de ciencia: una "ciencia que cuenta" y una "ciencia que no cuenta". Por cierto, es importante señalar aquí que la expresión "la ciencia que cuenta" no hace para nada referencia a su calidad, ya que simplemente es una valoración de "lo que importa" para "algunos" en "ciertas circunstancias". Esta distinción entre lo que cuenta y lo que no, corresponde a un sistema de priorización de las preguntas que las ciencias deben abordar. Este control estaba ampliamente autorizado por esta clase de instrumentos llamados revistas científicas centrales. Éste es el sistema de comunicación científica que comenzó a surgir de manera original en los años 19601970, en gran parte gracias a los efectos de lo que Eugene Garfield logró posicionar y vender como productos: el Journal Citation Reports y el Science Citation Index.

No hay intención maliciosa en Garfield. Mientras comete el error de razonamiento mencionado anteriormente, él simple y pragmáticamente busca 1) resolver un problema de seguimiento de citas y 2) respaldar la calidad de la respuesta que estaba en el proceso de ofrecer. Además de esto, Garfield también se encontraba en un contexto comercial y no era un académico puro ni mucho menos; más bien, tenía conocidos serios en este entorno y, en general, se sentía bastante cómodo con los académicos. Conocí a Garfield y mantuve de él el recuerdo de alguien que, más bien, se comportaba como un académico. En otras palabras, él tenía una pequeña empresa por administrar.

Los primeros estudios sobre citas fueron realizados por investigadores como Derek de Solla Price y por varios estudiantes de Robert Merton sobre la base de los 
resultados ofrecidos por la compañía de Garfield. Estos revelaron la existencia de regularidades en estas citas. Sobre la base de unas pocas miles de revistas, se desarrolló un nuevo campo de estudio: Scientometry. La revista Scientometrics se lanza, curiosamente, en un gesto de acercamiento entre los estadounidenses y los soviéticos y, por lo tanto, revela una cooperación este-oeste que pasa por Budapest. Pero quizás el efecto más importante de la cientometría emergente fue sentar las bases para una evaluación cuantitativa de las revistas científicas, para encontrar una manera de clasificar las revistas de acuerdo con la lógica aparentemente implacable de una métrica.

En este contexto surgió la cuestión de la "calidad de las revistas", acompañada de un malentendido, un doble malentendido de hecho. Por un lado, esta "evaluación" cuantitativa de las revistas pierde en gran medida su objetivo, ya que, en lugar de medir la calidad de las revistas, en realidad evalúa su visibilidad y prestigio. Además de esto, hay que tener en cuenta que dicha operación se realiza dentro de un club de revistas que han sido seleccionadas, como hemos visto, de manera rigurosa. El segundo elemento de malentendido es que, incluso si logramos enfocarnos en la calidad de las revistas, no tendría nada que ver con la calidad del trabajo científico contenido en ellas. ¿Qué significa la calidad de una revista? Es el trabajo científico lo que nos interesa, no la calidad de la revista. Cuando Mendel publicó sus trabajos sobre guisantes lisos y guisantes arrugados en el siglo XIX, lo hizo en una oscura revista de Moravia. Si bien la calidad del trabajo de Mendel no está en duda, lo que provocó el hecho anterior fue que el trabajo no fuera muy visible y, por lo tanto, haya sido difícil de detectar. Además, propuso tesis lo suficientemente nuevas e inquietantes que complicaron su recepción.

Gracias a las innovaciones de Garfield, empezamos a hablar sobre la calidad (a menudo confundida con el prestigio) de las revistas. Para la administración de la investigación, esta captación de investigadores por parte de las revistas fue una bendición. Recuerdo aquí las discusiones entre los cienciómetros y la administración de la Universidad de Montreal en la década de 1970. Para esta última, la aparición de un índice cuantitativo que supuestamente medía la calidad 
de las revistas científicas constituyó la posibilidad de una evaluación objetiva y cuantitativa y, por lo tanto, indiscutible. Su interés radicaba en el hecho de que los resultados de estas "mediciones" tenían la posibilidad de extenderse al trabajo de investigadores independientes. La aparición de la métrica del "factor de impacto" prometió, por lo tanto, evitar muchos problemas al evaluar los archivos de los investigadores que buscaban la tenencia, la promoción o el apoyo financiero. Finalmente se iba a tener una forma "objetiva" de evaluar a los investigadores, una perspectiva irresistible para cualquier administrador. Que este método de evaluación se haya extendido como un incendio forestal no debería sorprender a nadie.

Para los principales editores, esta evolución en la evaluación fue igual de positiva. Primero, Garfield logró imponer su sistema central de revistas, lo que significó que todas las bibliotecas pensaran que tenían que comprarlas y, por lo tanto, estaban listas para pagar casi cualquier cosa por ellas. Como consecuencia de esto, las grandes editoriales descubrieron que las revistas principales de Garfield eran un excelente mercado inelástico del cual podrían beneficiarse. De ahí que no fuera coincidencia que, después del éxito del Science Citation Index, el aumento meteórico en el precio de las revistas científicas comenzara a ser evidente. El desarrollo de la situación económica de las revistas científicas modernas, que todavía está presente, está vinculado con este sistema. Por lo tanto, la situación actual se basa en el doble malentendido mencionado anteriormente, ya que los administradores de la investigación quieren aprovechar la oportunidad para simplificar su trabajo, haciéndolo menos cuestionable e incluso reduciendo el costo de su evaluación. La razón de esto se debe a que es mucho más fácil ver rápidamente los factores de impacto de las revistas donde las personas publican que evaluar los artículos de estos investigadores individualmente. De igual manera, los editores ahora están en una competencia comercial bien regulada en donde su objetivo es simple: todo lo que tienen que hacer es aumentar el factor de impacto de sus revistas. 
La importancia atribuida al factor de impacto ha dado lugar al conocido sinsentido de los tres decimales en la expresión de esta métrica. Sobre este punto, pregunté directamente a Garfield hace muchos años y él respondió que realmente no necesitábamos tres decimales y que uno sería suficiente, pero sin justificar siquiera el porqué. Un decimal. ¿Por qué uno? ¿Por qué tres?

En una conferencia que Garfield dio alrededor de seis meses después de que lo cuestionara sobre este tema, dio una especie de respuesta a esta pregunta: Realmente no necesitamos tres decimales, escribe, pero si no tuviéramos tres decimales, no podríamos clasificar las revistas de forma "rigurosa". Riguroso, aquí, parece referirse a la falta de empate. La idea de tres decimales, por lo tanto, no consiste en aumentar la precisión de la medición, sino, más bien, en evitar un empate para intensificar la competencia entre revistas, situación requerida para operar un sistema donde los editores compiten para aumentar su cuota de mercado. Esto nos recuerda el problema de los organizadores de competencias deportivas: se quejaron de la medición de sprints en las décimas de segundo, dado que había demasiados récords mundiales en los cien metros planos. Se tuvo que hacer algo para relanzar la competencia y, especialmente, para captar el interés del público; se tuvo que ir a la centésima de segundo, incluso si esta pseudo-precisión no tuviera ningún sentido atlético.

Muchas personas involucradas en la política científica están convencidas de que la actividad científica debe ser gestionada a través de la competencia. A menudo, esta actitud se basa en argumentos tomados de la teoría de Darwin. Cuando la situación de la selección natural de Darwin entra en juego, la competencia funciona desde abajo, no desde arriba. Tome el ejemplo de un león que quiere una buena comida de gacelas. Lo que la teoría de Darwin dice no es "encontrar la gacela más rápida"; por el contrario, dice "encontrar la gacela más lenta". Se eliminará tal gacela que, por lentitud o mala suerte, no pueda escapar del león. Si adoptamos el punto de vista evolutivo correctamente en la ciencia, tendríamos que buscar un sistema que pudiera usarse para eliminar a los investigadores menos interesantes de la investigación. Pero el discurso dominante dice todo lo contrario: hay que buscar a 
los campeones, reclamar la excelencia y pretender que cada investigador es un campeón potencial.

Todo esto conlleva a una situación que pervierte el sentido de evaluación de los investigadores y la investigación: los investigadores son evaluados a través de revistas erigidas como monumentos de calidad, mientras que la competencia entre revistas se juega realmente en términos de visibilidad y prestigio. Esto también equivale a decir que, de manera silenciosa, los grandes laboratorios y líderes de la ciencia en los grandes países del mundo han establecido una especie de política científica colectiva y tácita que, de hecho, determina a través del factor de impacto la competencia entre revistas, las preguntas que importan y las preguntas que no.

De hecho, tenemos un muy buen ejemplo indirecto de esta política tácita de las ciencias con el caso de este médico de Calcuta, Shambhu Nath Dhe, considerado por algunos como no creíble. Después de una investigación excepcional, ayudó a renovar nuestro conocimiento sobre el cólera, al llamar la atención de los investigadores sobre sus enterotoxinas. También logró hacerse escuchar por colegas ingleses y estadounidenses, lo que le dio un acceso excepcional a la revista Nature. A pesar de contar con la ventaja de publicar en esta revista, su artículo se descuida. Nadie lo cita por 15 o 20 años. La razón es que Dhe, en un país casi desconocido del Tercer Mundo, aborda un tema que no se percibe como un frente "caliente" de la ciencia. Garfield incluso escribió un artículo sobre este ejemplo, para tratar de entender lo que había sucedido según las citas de este artículo. Su conclusión, bastante desalentadora en la banalidad, es que la falta de interés en el trabajo del Dr. Dhe corresponde claramente a "un caso obvio de reconocimiento tardío". Molière y su virtud latente de las pastillas para dormir lo hubieran apreciado.

\section{El surgimiento de la tecnología digital y algunos de sus efectos}

Entremos ahora en la cuarta y última fase de la historia de las revistas científicas, la

cual está marcada por la llegada de la era digital. Las nuevas tecnologías han cambiado y continúan cambiando toda una serie de prácticas, procesos y también 
relaciones de poder dentro de todos los actores involucrados en la comunicación científica. Cuando lo digital comienza a aparecer, ocurren dos fenómenos: por un lado, estamos presenciando exploraciones y experimentos de investigadores que hacen uso de nuevos medios (yo fui parte de ese hecho, ya que con unos colegas creamos una revista académica que comenzó en 1991 y que duró 10 años: Superficies). Para estos investigadores, lo digital era una forma de superar un cuello de botella en la comunicación académica, un obstáculo relacionado con el hecho de que, para lanzar una revista impresa, era necesario tener mucho dinero y tiempo. En la práctica, sólo las editoriales podrían crear nuevas publicaciones científicas impresas. Sólo ellas podían invertir 7, 8 o 10 años en una revista para permitirle alcanzar una cierta visibilidad y, como consecuencia, un punto de equilibrio. Varios investigadores comenzaron a publicar revistas académicas electrónicas (la nuestra fue la décima en aparecer en el mundo), tratando de romper este cuello de botella y demostrando que los investigadores podían, en cierta medida, asumir el control de los medios de comunicación que necesitaban.

Sin embargo, estos investigadores lanzaron revistas que conservaban la forma tradicional de estos dispositivos. La mayoría de nosotros, en esos años pioneros, mirábamos hacia el futuro como si fuera un espejo retrovisor. Haciendo uso de la famosa fórmula de Marshall McLuhan, lo que estábamos tratando de hacer era, simplemente, transponer una revista clásica en formato digital. Sin embargo, al tratar de desarrollar este nuevo dispositivo, nosotros, como todos, aprendimos mucho.

Las editoriales comerciales también comenzaron a explorar lo que podrían obtener de lo digital. Es interesante revisar las primeras ideas para la publicación digital de ciertas editoriales, como Elsevier. La editorial Elsevier, que existe hoy en día, es una compañía creada en 1880. Hasta la Segunda Guerra Mundial, era sólo una pequeña compañía que buscaba cumplir algunos de los roles que describí anteriormente hablando de vendedores entre 1830 y 1945. En 1991, Elsevier, que luego se convirtió en un actor importante de la publicación científica, decidió explorar las posibilidades de lo digital. Su estrategia inicial era instructiva, divertida en 
algunos aspectos, pero también extremadamente mañosa por otro. Como parte de ésta, Elsevier se compromete a digitalizar algunas de las revistas científicas que controla, para lo que esta editorial imagina vender estas revistas en forma de videodiscos a las bibliotecas. Para proteger su propiedad intelectual, Elsevier juega con el hecho de que el tipo de formato y el tipo de ancho de banda que se necesita para leer estas revistas requieren que los discos de video sean consultados en el sitio, en la biblioteca. En resumen, lo digital comienza en Elsevier con la idea de reemplazar el papel por videodiscos, tal como lo habríamos hecho con las microfichas. La idea de jugar con un ancho de banda bajo podría compararse con la imposición de un control sobre los lectores de microfichas. El razonamiento no era del todo incorrecto, pero estaba incompleto. De hecho, Elsevier comprendió rápidamente que la muralla del bajo ancho de banda sólo iba a durar un tiempo, probablemente muy corto, con el riesgo de ver al pájaro digital salir volando de su jaula temporal.

Sin embargo, la primera experiencia de Elsevier en la publicación de revistas electrónicas no se limitó a nuevos medios de difusión. De manera más fundamental y radical, Elsevier también se comprometió a revisar a fondo los marcos legales para sus transacciones con las bibliotecas. En el proyecto de Elsevier, conocido por el nombre holandés TULIP, se incorpora la letra $L$ a su acrónimo, que se refiere a la palabra Licencia. Es este en verdad un detalle importante, ya que influirá en el resto de la historia de las revistas científicas.

De hecho, Elsevier comprende que con lo digital ya no se venden objetos. Si se vende algo, es acceso, pero nunca se vende a lo que le das acceso, ya que se firman contratos que dan acceso al contenido del cual uno conserva la propiedad total. De este modo, Elsevier comienza a desarrollar, en forma digital, una manera de controlar el contenido de las publicaciones académicas que irá mucho más allá de lo que la impresión y los derechos de autor habían establecido. Por ejemplo, una vez que una revista impresa se vende a una biblioteca, los derechos de propiedad se agotaron de acuerdo con los derechos de autor o de las leyes de derechos de autor. Como resultado, la biblioteca podría hacer casi todo lo que quisiera con esta 
revista, incluso prestarla a las personas y permitirles verla libremente, sin que ellas mismas la compraran. El desafío para Elsevier, al controlar el acceso a través de un acuerdo de licencia, era revisar a fondo ciertos derechos disponibles para los compradores de materiales impresos, incluso sujetos a derechos de autor. De ahí que empezara a surgir un mundo completamente nuevo, en el que la biblioteca perdía la propiedad de la actividad a la que se dedicaba.

En esta revolución silenciosa, se llevaron a cabo, en el marco de lo legal, las negociaciones entre bibliotecas y editoriales que condujeron a transformar, e incluso revertir, el papel de los primeras. Originalmente, abierta a las personas para ofrecerles la posibilidad de consultar documentos impresos, la biblioteca se convirtió en "aduana" al requerir la identidad de la persona que busca acceso a una revista para verificar si tiene derecho a hacerlo o no.

Estos cambios aún están en curso y a veces tienen consecuencias sorprendentes. Por ejemplo, un proceso legal acaba de comenzar entre Elsevier y la Universidad del Estado de Louisiana, en los Estados Unidos. ¿Por qué? Debido a que las negociaciones entre esta universidad y Elsevier no marchaban completamente a favor de Elsevier, esta editorial redujo el perímetro de accesibilidad a sus revistas. En otras palabras: "Aceptamos los términos de su contrato, siempre que usted acepte la exclusión de ciertas unidades o subsidiarias de su institución". La Universidad del Estado de Louisiana decidió probar la legalidad del proceso con un tribunal de justicia. Dicho esto, la universidad ahora debe confiar en la ley holandesa y no en la del estado de Louisiana.

Fue con la tecnología digital como los editores comenzaron a implementar una estrategia de marketing conocida como Big Deal. Tradicionalmente, las bibliotecas habían establecido la lista de títulos de revistas deseadas en función de las líneas de investigación de su institución. Considere una universidad que quiere acceder a alrededor de 300 revistas de la colección de 1,500 revistas de una editorial internacional. Digamos que esta editorial ofrece un precio tal que la biblioteca sólo puede permitirse comprar acceso a 250-270 títulos por un costo total que fácilmente puede rondar el millón de dólares. Pero la editorial también presenta una oferta 
complementaria: el resto del catálogo por solo 100,000 o 150,000 dólares más. El resto del catálogo no es de gran importancia para la biblioteca, porque la gente, localmente, probablemente no va a usar estas publicaciones periódicas pero, obviamente, el precio por título caerá considerablemente un argumento importante para un agente financiero de la institución. Dicho esto, lo que le importa a la biblioteca no es el precio por título de revista, sino el precio por consulta de artículo.

Las bibliotecas tienen presupuestos limitados. Si, debido al Big Deal, se les pide que paguen un 15\% más por cada editorial grande, el margen de maniobra presupuestario para las otras editoriales se reduce aún más. Con la reducción de los mercados, estas editoriales y muchas sociedades científicas ya no pueden mantener vivas sus revistas. En este contexto, las grandes editoriales pueden ofrecer ayuda a las pequeñas, comprándoles títulos y ofreciéndoles tomar posesión de ellos, aunque dejándoles a cargo del contenido. Excepto que, si surgen diferencias entre el comité editorial y la gran editorial, esta última probablemente tendrá los medios para imponer sus puntos de vista sobre el comité editorial. Gracias a la tecnología digital, las grandes editoriales pueden influir mejor en el funcionamiento y en las instrucciones de la publicación científica. Este poder no está organizado de manera central, visible o sistemática, pero está disponible. Esto es lo que a veces percibimos cuando un comité editorial claudica por unanimidad y trata de relanzar una nueva revista, a menudo con la obligación de usar un nuevo título, además de todos los problemas que conlleva recrear una identidad, reputación y visibilidad para la nueva revista.

También debe tenerse en cuenta que, en formato digital, navegamos por los textos de manera diferente. Más allá de los motores de búsqueda, también existen plataformas dentro de las cuales las revistas viven de los medios para atraer a los lectores a consultar otras revistas alojadas en la misma plataforma. Estos sistemas de atracción web, estrategias inicialmente desarrolladas por sitios pornográficos, se basan en las leyes de una economía de atención: cuanto más tiempo podamos mantener a las personas en casa, menos tiempo pasarán con otros. $Y$ lo más 
importante, al leer más nuestras revistas, citarán algunas, lo que mejorará aún más el factor de impacto.

Así es como aparecieron rápidamente herramientas que resultaron útiles pero que también tuvieron efectos secundarios significativos; por ejemplo, en Science Direct, la plataforma de Elsevier, donde el artículo que uno está leyendo ha sido citado en otros artículos también ubicados en Science Direct, lo que atrae a un investigador a querer rastrear toda una hipótesis o teoría.

Por lo tanto, la revista tiene lugar dentro de un todo mucho más grande y complejo que, de hecho, es un dispositivo para la circulación del conocimiento. Según Olivier Ertzscheid, este nuevo tipo de dispositivo de publicación contiene lo que cualquier plataforma posee, es decir, un "régimen de verdad". ${ }^{4}$ Aplicado por Ertzscheid a las plataformas de Google, Facebook y Wikipedia, este razonamiento también puede arrojar a la luz el comportamiento de las plataformas de búsqueda, sobre todo porque el "régimen de verdad" de Google se basa en un algoritmo del "rango de página" que está directamente inspirado en la estrategia de Garfield. Sin embargo, este régimen de verdad se caracteriza, sobre todo, por la reunión entre lo "verdadero" y lo "popular", así como en el campo de las publicaciones científicas descubrimos que el "valor" de las publicaciones científicas corresponde, de hecho, a su visibilidad y prestigio, elementos que tienen alguna relación con la idea de popularidad. Google aplica este principio directamente en Google Scholar, por lo que las grandes editoriales están adaptando el comportamiento de sus propias plataformas para aumentar la visibilidad de sus productos.

En esta transformación, podemos notar que el papel de la revista evoluciona de manera marcada, particularmente al establecer una relación jerárquica entre la revista y la plataforma correspondiente. La lógica de esta última tiende a sobresalir de la propia de la revista científica, que, de todos modos, se convirtió en la tercera y cuarta fase de su historia. Esta tendencia se confirmará cuando aparezca la invención de lo que a menudo se llama una "mega-revista". Con Plos One, inventada alrededor del 2008, estamos en el proceso de cambiar a un sistema de comunicación científica fundamentalmente nuevo. De hecho, con tal plataforma, la 
cuestión de la orientación editorial ya no tiene mucho significado. Cuando consideramos que la identidad de una revista está fuertemente vinculada con su orientación editorial, ver que se desvanece corresponde a un cambio profundo en el tema de la revista; en particular, los métodos para evaluar los resultados de la investigación ya no se refieren solo a la calidad del trabajo científico, ya que ahora los pares examinan las metodologías, la calidad de las observaciones, la calidad de los experimentos, todo lo relacionado con el método científico y la forma de crear conocimiento científico. Por otro lado, las preguntas de "relevancia" de esta investigación se eliminan completa o ampliamente, independientemente del significado asignado en cada revista a la "relevancia".

Con lo digital, todo puede ser accesible desde cualquier lugar. En ausencia de barreras financieras como la tradicional suscripción, podemos esperar volver a conectarnos con la lógica de la comunicación científica tal como existía en el momento de la correspondencia entre los científicos. Esto lleva directamente al concepto de "acceso libre". De hecho, todos los experimentos en revistas electrónicas del período 1988-1995 fueron revividos a nivel social e institucional por la Iniciativa de Acceso Abierto de Budapest de 2002 y declaraciones posteriores (Berlín, Bethesda, etc.). De esta serie de iniciativas nació la idea del libre acceso en forma de movimiento internacional.

El advenimiento del acceso abierto también ha ayudado a desafiar los pilares financieros tradicionales de la publicación científica, de ahí que se hayan inventado nuevas estrategias comerciales, algunas de las cuales han sido adoptadas por grandes editoriales comerciales. De hecho, ha comenzado a preocuparse de que el sistema de suscripción imponga límites a los ingresos. Los presupuestos de adquisición de bibliotecas tienden a estancarse o a crecer demasiado lento para su gusto. Como consecuencia, encontrar fuentes alternativas de ingresos parece importante para estas grandes empresas. Identificar nuevas fuentes de ingresos, como las instituciones que financian la investigación, se ha convertido en un ejercicio importante para las editoriales. Al mismo tiempo, se están desarrollando productos mixtos, como revistas híbridas que permiten a las revistas tradicionales 
alojar artículos de acceso abierto. Presentadas como un medio para garantizar una transición sin problemas hacia el acceso abierto, las revistas híbridas, sobre todo, permiten la creación de situaciones vagas y duraderas, organizadas en torno a un doble sistema de ingresos: suscripciones por bibliotecas y pago de "cargos por procesamiento de artículos" para los autores o sus sustitutos. En resumen, toda una serie de cambios financieros indica que los objetos clásicos de la comunicación científica están cambiando.

Paralelamente a estas tendencias, hay otro lado del acceso abierto que se llama Green y que se basa en el autoarchivado de las publicaciones de los investigadores en "repositorios institucionales". Esta forma "verde" de acceso abierto fue ampliamente conceptualizada y defendida por mi colega de la Universidad de Quebec, en Montreal, Stevan Harnad. Green es un elemento fundamental en la ecuación general de acceso abierto. Obviamente, las editoriales estaban preocupadas por el estado de la situación e intentaron, más o menos abiertamente, más o menos directamente, oponerse a esta estrategia. Las reglas de depósitos fluctuantes o los requisitos de formato variable son sólo algunas de las artimañas utilizadas por algunas editoriales para empeorar las cosas y hacer que todo sea más confuso. Al mismo tiempo, la versión "verde" del acceso abierto planteó la cuestión de la versión de referencia de los resultados de búsqueda. Si la editorial, para no golpear a los investigadores de frente, acepta el depósito de algo que se parece a la versión publicada en una revista, pero que no es exactamente lo mismo, ¿cómo distingue otra "versión" de la presentada como versión oficial? Y si la diferencia es puramente en apariencia, en forma, ¿por qué no citar directamente a la que está bien archivada en una buena universidad y que es completamente accesible?

De la cuestión de las versiones nació otra pregunta más profunda: ¿cómo lidiar con las diferentes versiones de los productos de investigación? Más bien, ¿éstas no corresponden con la evolución natural de un documento de trabajo sujeto a preguntas, críticas y comentarios de colegas? Y si es así, ¿cuál es el significado de un artículo científico? Esta pregunta se refiere a la cuestión de los "cristales del conocimiento" que desarrollé en un intercambio con Thomas Wiben Jensen, 
publicado en un artículo reciente. ${ }^{5}$ En este debate con Jensen, que quería defender una revista de lingüística mientras estaba muy preocupado por la supervivencia de la suya en el régimen del acceso abierto, cuestioné la necesidad misma de una revista y la de los artículos. Hice la pregunta: ¿qué necesitan los investigadores en última instancia? Necesitan buenas personas con quienes hablar. Además, cuando trato de interrogar a otra persona sobre un trabajo, lo que necesito no es un artículo presentado como la forma final, sino un documento de trabajo suficientemente estable para permitir que comience un diálogo. Si este documento de trabajo estable se llama o no un "artículo" es de poca importancia, lo importante es la presencia de una herramienta de comunicación que permita mantener una discusión regulada sobre un tema. Llegamos así a una visión fluida de los frutos de la investigación como un medio para apoyar el diálogo entre investigadores. Con esta perspectiva, ya no necesitamos revistas centrales o clasificaciones de revistas, sino que debemos pensar en cómo reconocernos y cómo identificarnos, sin necesariamente pasar por los sistemas de prestigio y visibilidad que se construyen, sobre todo, con el doble propósito de facilitar la producción de conocimiento e intensificar la generación de ganancias.

Creo que una de las grandes preguntas que se resolverá en los próximos años es precisamente el modelo fundamental de publicación que debe estar en el corazón de lo que he llamado "la gran conversación científica". Para ello, creo que el modelo más claro, y al mismo tiempo el más convincente para concebir la posible forma de este futuro, será el del software libre. Los programadores, en el contexto del software libre, han aprendido a contribuir individualmente mientras se posicionan en una relación de repartición distribuida y deslocalizada. Aprendieron a contribuir de manera aditiva a un proyecto común que puede ser un software muy simple o, por el contrario, un software muy complejo. Aprendieron a administrar estas contribuciones a través de un sistema de versiones bien regulado. Me parece que el desafío de las publicaciones académicas de hoy en día es precisamente la creación de un modelo controlado y regulado por el flujo de las conversaciones, para lo cual el modelo del software libre me parece absolutamente decisivo y 
completamente obvio, para mí, de pensar e imaginar como futuro de la publicación académica.

Mientras tanto, es probable que la comunicación de la investigación vea un aumento en la importancia de las redes y plataformas que trabajarán juntas. Estamos viendo, por ejemplo, redes de repositorios institucionales como OpenAIRE, en Europa; La Referencia, en América Latina, y otras más que demuestran que esta tendencia se está extendiendo en todo el mundo. También debemos señalar la existencia de plataformas como Revues.org, en Francia, que están desarrollando libremente lo que las grandes editoriales están construyendo de una manera más o menos cerrada. A través de estas tendencias, lo que veo emergente es la posibilidad de imaginar que las comunicaciones científicas se organicen en términos de la constitución de "territorios científicos". Para avanzar un poco más en lo que quiero decir con esto, confío en la noción de los geógrafos que definen un territorio como un espacio estructurado mediante la comunicación. Cuando los individuos, los investigadores, deciden comunicarse entre sí para resolver un problema, confían en medios de comunicación que se mantendrán estables durante cierto tiempo y que conducirán ipso facto a la definición de un territorio. Por lo tanto, un territorio científico, incluso si es temporal, permanecerá en actividad mientras la cuestión científica siga viva y los medios de comunicación sean estables.

Si tuviera que dar como conclusión una especie de etiqueta sobre lo que podría ser el futuro de la revista, sería precisamente su mutación en el micro-territorio gestionado por las plataformas entrecruzadas, sin olvidar el hecho fundamental de que una "revista" se convierte en un medio de expresión para una comunidad, aspecto que revive de las revistas de las sociedades científicas en el siglo XIX.

\section{Debate con Jean-Claude Guédon}

Alain Loute: Una de las cosas que me interesó de su trabajo es que, cuando habla de acceso abierto, siempre lo incluye en lo que es para usted el espíritu de la investigación científica que califica esta expresión de "la gran conversación 
científica". Esto me parece interesante porque nos permite no sólo entender el tema del acceso abierto simplemente como acceso libre y abierto, sino también los productos particulares de la literatura científica.

Jean-Claude Guédon: Y que nos hacen prisioneros de objetos tradicionales, como la revista desde la Segunda Guerra Mundial, precisamente, o el artículo en sí.

A. L.: Es interesante porque esto hace referencia a una fuerte noción de acceso; acceso a una forma de práctica más que a unos bienes. Una "práctica", por lo tanto, en el sentido de la comunicación, pero también en el sentido de la cooperación. En su trabajo, cuestiona un poco la imagen romántica del autor individual y retoma la imagen del "sistema de inteligencia distribuida", particularmente, en el intercambio con Jensen. De ahí que el acceso abierto deba significar el acceso a una comunicación y a una práctica cooperativa. En cierto modo, es la imagen de la participación en una ciudad del conocimiento como conversación y cooperación.

J.-C. G.: Absolutamente. Incluso, iría más lejos; diría que, si los seres humanos, usando un término general, se dan cuenta de la importancia de exceder las capacidades de la inteligencia individual, es precisamente debido a este tipo de proceso de comunicación. De hecho, la "revolución cognitiva", de la que los antropólogos y arqueólogos hablan sobre la humanidad, a menudo se percibe como un efecto del desarrollo de un lenguaje, que puede ir más allá de la realidad concreta para alcanzar el concepto y/o lo imaginario. Esto es la primera etapa de una evolución colectiva que luego lleva a la escritura y luego a la Revolución Científica, concebida aquí como la elaboración de una forma distribuida de comunicación regulada entre socios mutuamente reconocidos: los "pares".

A. L.: En el fondo, tal vez hubo un actor político, al menos en el tercero y cuarto periodo, que pudo haber mencionado menos. Lo pone muy bien en el segundo período al mostrar cómo las sociedades científicas y las academias también tuvieron que gestionar su relación con las autoridades políticas. 
J.-C. G: Pero también aparece en el tercer periodo, me parece a mí, con los editores comerciales, lo que demuestra que lo que ha llamado el actor político se está retirando gradualmente del campo de la publicación científica para centrarse en la financiación de la investigación propiamente dicha. El actor político en cuestión, tal vez en busca de la simplificación de su comportamiento y de las cuentas mal calculadas, ha permitido el desarrollo de un gran poder comercial que no sólo les cuesta a las instituciones públicas o privadas, sino que influencia, indirectamente y en silencio, la orientación y el significado de la producción de conocimiento.

Tal vez debería haber hablado sobre el aumento de la investigación científica después de la Segunda Guerra Mundial. Aquí es cuando debería hacer mención del nombre de Robert Maxwell, ya que él vio cómo importar competencia de tipo comercial a un campo que se había alejado implícitamente de este tipo de comportamiento. Esto lo consiguió introduciendo, en todos los campos, revistas "internacionales" capaces de publicar rápidamente y bastante bien, abandonando el costo de páginas adicionales, y el de las ilustraciones, para atraer a autores conocidos (y trasmitiendo los costos a las bibliotecas), criticando la atmósfera de los exclusivos grupos que dominaban la selección de artículos en los equipos editoriales, e inventando la revisión por pares en formas aparentemente más objetivas; en resumen, modificando el proceso de producción ( $\mathrm{y}$, por lo tanto, el significado) de un diario académico. Con esto, Maxwell demostró que el comercio pudo entrar en este campo con éxito.

Además, no debemos olvidar que tener una reconocida plataforma para la publicación científica significa tener un dispositivo panóptico extraordinariamente poderoso para la investigación científica. ¿Qué se puede decir sobre un laboratorio cuando se tiene una visión bastante buena de lo que se lee en este laboratorio? Raramente hablamos de estas cosas, pero debe recordarse que Maxwell frecuentaba los servicios secretos británicos durante la Segunda Guerra Mundial. 
En general, las autoridades de la ciencia, en particular las academias y las sociedades científicas, no sabían cómo reaccionar ante las nuevas demandas de una investigación científica fuertemente acelerada. Las empresas comerciales, por el contrario, encontraron una manera de ingresar al juego de las publicaciones y, una vez que ingresaron, comenzaron a trabajar en la dirección de la producción de conocimiento científico para adaptarla a las necesidades de una situación que se había vuelto comercial. Así es como la búsqueda de la excelencia, el énfasis en la competencia (prácticamente excluyendo la cooperación) y el cambio de la calidad del trabajo por la calidad de la revista (este último aspecto que se asimila, más bien, a la visibilidad y al prestigio) han contribuido a dar un nuevo significado a los productos de investigación y a la producción de conocimiento.

A. L.: Veamos más específicamente el cuarto periodo. Usted subrayó el hecho de que el ideal de acceso abierto, incluso antes de la iniciativa de Budapest, fue conducido por los mismos investigadores que, como usted dijo, experimentarán con nuevas formas de revisión utilizando lo digital como medio para reclamar estas formas. ¿Este ideal de acceso abierto conducido por estos investigadores, y que encaja perfectamente con lo que usted llama el ethos de la gran conversación científica, es lo mismo o es probable que entre en tensión con el ideal de acceso abierto como lo usan los actores políticos, ya sea por los Estados Unidos, Gran Bretaña o por la recomendación europea de 2012? Planteo este punto porque, en la literatura sobre el tema, el discurso político sobre el acceso abierto plantea preguntas y acertijos con los académicos y el mundo editorial. Pienso, por ejemplo, en los planteados por Philippe Minard, ${ }^{6}$ secretario del consejo editorial de la Revue d'Histoire Moderne et Contemporain, o por Ghislaine Chartron. ${ }^{7}$ De ahí que se haya debatido en los campos de las revistas de humanidades y ciencias sociales, dos campos con posiciones opuestas y claras, sobre la situación de Francia, particularmente en 2013. ${ }^{8}$ Para algunos, el discurso político del acceso abierto está motivado principalmente por el deseo de establecer instrumentos de gestión y evaluación de la investigación a través, 
y particularmente, desde una posición panóptica, con el fin de imponer sus términos y con lo cual los archivos abiertos y los portales agregados proporcionarían acceso. ¿Qué piensa usted?

J.-C. G.: De hecho, en ocasiones tuve debates algo difíciles con Ghislaine Chartron. Ella parte de los axiomas básicos, que son que 1) los investigadores son incapaces de manejarse a sí mismos en lo que respecta a la comunicación científica; requieren, dice, mediación, un término muy general y algo difícil de ser identificado, especialmente con la fuerte presencia de mecanismos de revisión por pares administrados por equipos editoriales que generalmente están compuestos por pares; y 2) que el acceso abierto plantea riesgos para una estructura de comunicación científica comprobada. Este argumento pudo haberse aplicado a los impresores que amenazaron a los scriptoria al final del Renacimiento. El desafío fundamental, para ella, es preservar el sistema, o permitir que evolucione con seguridad, en lugar de pensar en la comunicación científica como una base esencial de la reflexión a realizar. Además, se posiciona resueltamente del lado de la perspectiva de las publicaciones científicas, en gran parte de humanidades, bajo el control de pequeñas editoriales francesas. Desafortunadamente, el combate particular de estas editoriales parece un poco periférico y representativo de las fuertes tendencias en el campo. Para mí, es como si, en el siglo XV, después de Gutenberg, quisiéramos evolucionar los scriptoria hacia la imprenta, lo cual me parecer ser un mal debate.

Sin embargo, éste es el debate que los grandes editores han abordado de cierta manera cuando hablan de sostenibilidad. Dicen a los políticos, por ejemplo, que no hay sostenibilidad fuera de un sistema de comercio, lo que implica que sean los únicos capaces de resistir sin aportes del sector público. Obviamente, esta tesis se basa en la presencia implícita y continua de presupuestos crecientes en una serie de instituciones públicas, ya que éstas contribuyen enormemente a la recaudación de ingresos por parte de las principales editoriales. 
Con este tipo de argumento, estamos equilibrando aquí, por un lado, la comunicación entre investigadores y las necesidades de una producción optimizada de conocimiento; y por el otro, el mantenimiento de las estructuras tradicionales de comunicación entre investigadores controlados, en gran parte, por grandes editoriales.

Personalmente, creo que lo digital, en cualquier caso, ya está cambiando el papel de las bibliotecas, el papel de las universidades, el de los centros de investigación, el de los organismos que apoyan y subvencionan la investigación, ya sean ministerios o grandes organizaciones sin fines de lucro, como Wellcome, como Hughes, etc. En resumen, los intentos de mantener nuestros homólogos de scriptoria están condenados al fracaso.

En cuanto a la cuestión de la evaluación, o incluso el seguimiento, debe recordarse, sin embargo, que los esfuerzos para establecer un régimen de evaluación basado en la clasificación de las revistas y el factor de impacto preceden al menos entre 25 y 30 años. Cuando, gracias al acceso abierto, comenzamos a imaginar formas de lectura asistida por computadora ("minería de texto y de datos"), y cuando, gracias también a éste, imaginamos publicar estadísticas sobre el uso de documentos almacenados en un repositorio institucional, se expresó el miedo a la vigilancia. Este miedo a la vigilancia es legítimo, y tiene sus raíces en las tradiciones bibliotecarias: cuando un bibliotecario no quiere revelar lo que un usuario pudo haber leído, recuerda las condiciones necesarias para la libertad de pensar, de ser informado y expresarse. Pero este miedo debe distinguirse analíticamente de la manía de la evaluación cuantitativa. En otras palabras, el acceso abierto no busca favorecer la llegada de ningún dispositivo panóptico, ya que la evaluación de la investigación ya había revelado un gran talento para crear procedimientos delicados mucho antes del surgimiento de este. Para ir más rápido, los tres decimales del factor de impacto, recordemos una vez más, preceden al acceso abierto por lo menos veinte años. De hecho, lo que debe cuestionarse es el objetivo político que intenta hacerse del acceso abierto, 
cuando el sujeto es verdaderamente el de la evaluación de la investigación y los investigadores. ¿Debería verse esto como un intento de asustar a los investigadores para mantenerlos alejados del acceso abierto, a pesar de las ventajas que obviamente tiene para la gran conversación científica?

A. L.: Esta vez tengo otra pregunta sobre el final de su presentación del día de hoy. Usted dijo que se deben inventar nuevas formas de estabilidad que permitan el diálogo, considerando que, fundamentalmente, las formas que se han estabilizado en el pasado no deben ser fetichizadas.

J.-C. G.: Realmente me gusta el término fetiche. Esto es exactamente lo que estamos condenados a hacer ahora.

A. L.: Esto último también hace eco de otro término que usa: momentos congelados. ${ }^{9}$ Para usted, no se trata de cuestionar esta idea del momento de estabilización. De igual manera, la noción de territorio es interesante en este punto.

J.-C. G.: De hecho, no es el artículo como forma lo que cuenta, es la idea de que varios investigadores acuerden considerar un resultado de investigación como temporalmente estable para examinarlo y trabajar en él con profundidad (y en común). Por cierto, el modelo institucional más obvio es el del seminario: llegas a un seminario, eres la persona a cargo del seminario esa semana. Presentas un texto con un poco de antelación, la sala reacciona, critica, responde, etc. Al final de esto, uno vuelve a trabajar en su texto y la fluidez comienza de nuevo. ¡Necesitamos de los seminarios! El seminario es también un espacio que de repente adquiere sentido al estructurarse a través de los canales de comunicación que se han establecido entre los participantes. Como resultado, se convierte en un territorio durante toda la sesión.

A. L.: Mi pregunta sería la siguiente. Mantener viva esta comunicación también es capaz de garantizar formas de memoria de estos territorios en particular. Esto me hace pensar en una reflexión de Roger Chartier $^{10}$ sobre las misiones de 
la patrimonialización de las bibliotecas, señalando que uno de los peligros sería creer que un texto es el mismo independientemente de su medio y que el formato digital de un texto podría reemplazar las otras formas que ha tomado en el tiempo. Robert Darnton menciona, en su Apologie du libre, ${ }^{11}$ este ejemplo de universidades estadounidenses que destruyeron publicaciones periódicas en formato de papel al conservar sólo una copia de microfilm. Para él, a través de estas destrucciones perdimos algo: el hecho de que la experiencia que tenemos de un texto en cierto formato no es exactamente la misma a través de otros formatos. Ésta es también la razón por la cual la cuestión de los formatos es una pregunta fuerte. No se trata sólo del equilibrio de poder con las editoriales que desean establecerse como garantes del único formato legítimo, sino que también se trata de hacer un seguimiento del hecho de que dicho artículo fue registrado en un entorno de preguntas, bajo cierto tema y en un determinado territorio. ¿Cómo hacer un seguimiento de todo esto? En mi opinión, el autoarchivado no lo garantiza.

J.-C. G.: Estoy de acuerdo con usted. Pero tenga cuidado de no confundir la versión con el formato. Ambos términos cuentan. También cabe señalarse que la transición a un régimen de licencia de acceso para documentos digitales deja la responsabilidad de los activos en manos de las editoriales. Nada es más efímero que una empresa privada, lo que probablemente explica su obsesiva relación con la "sostenibilidad". Por lo tanto, debemos pensar en soluciones a largo plazo, algunas de las cuales están empezando a surgir. La Biblioteca Real de Holanda tiene acuerdos de tipo patrimonial con Elsevier. El proyecto Lockss, lots of copes keep the stuff safe, de la Universidad de Stanford, también aborda este importante problema.

A. L.: Quizás, a nivel técnico, puedan existir soluciones, pero creo que sería un engaño creer que, por sí sólo, el depósito simple podría garantizar la memoria de todos los rastros.

J.-C. G.: El depósito simple no es suficiente, es obvio. Un documento sólo vive si una comunidad lo alimenta permanentemente. Abandonado, sólo parece 
cifrado, indescifrable. $Y$ es por eso por lo que las redes de repositorios institucionales y los servicios que se están construyendo a su alrededor son extremadamente importantes. Me gustaría volver aquí a la figura del software libre para el lado patrimonial de esta pregunta. Creo que lo que será extremadamente importante es contar con menos colecciones de revistas, como las tenemos actualmente, sino con más colecciones de versiones. Versiones que hablarían de "momentos congelados", es decir, de estos momentos del seminario, donde cada vez hay algo que se ha estabilizado un poco más, en un momento dado, y que luego continúa. Considere también el ejemplo de Wikipedia. Uno puede hacer un cierto juicio, ciertamente no completo pero instructivo, en las páginas de Wikipedia al estudiar la historia de la página. Vemos que se está desarrollando un consenso relativo y temporal en torno a un tema, y la intensidad de los intercambios constituye una especie de posible indicador indirecto de la calidad del resultado actual. Creo que hay una importante lección detrás de Wikipedia y detrás de su "régimen de verdad", que Olivier Ertzscheid analizó tan bien en su seminario mencionado anteriormente. Si podemos mantener todas las versiones y todos los debates que acompañaron el paso de una versión a otra, y así sucesivamente, estamos en el proceso de establecer una memoria general del esfuerzo intelectual de los seres humanos, que va mucho más allá de lo que hemos podido hacer hasta ahora. Agregue a esto lo que aún no hemos hablado aquí pero que se ha hecho posible gracias a la tecnología digital, es decir, el hecho de que en la elaboración de un artículo, en el sentido clásico del término, tomamos conciencia de que también hay toda una serie de actividades subyacentes importantes: producción de datos, ordenamiento de datos, elección de datos, interpretación de datos, etc. Al revelar el andamiaje fáctico y observacional de cualquier momento congelado, nos damos cuenta de que, sin estos elementos, el artículo científico actual (o sus sucesores) pierde gran parte de su significado. ¿Cómo podemos afirmar que el experimento científico puede repetirse y así confirmar la exactitud del avance, si no tenemos los datos iniciales? Por supuesto, con los datos modernos se 
necesita un software para procesarlos, que debe ponerlos en una forma que permita su reutilización más allá del alcance inicial de su aplicación; pero eso nos llevaría demasiado lejos. Éste es el trabajo, por ejemplo, de la Alianza de Datos de Investigación. Estamos comenzando a ver el nacimiento del momento en que tendremos los medios técnicos para poder recuperar datos, encontrarlos, clasificarlos, reorganizarlos, etc. Entonces, podremos crear nuevos territorios resaltando las congruencias que no son aparentes a priori pero que nos permitirán intervenir para decir: "¡Aquí hay preguntas interesantes!”, “Qué estás haciendo con eso?”, etc. Un aspecto nuevo y emocionante en todo esto es que las máquinas también podrán intervenir.

Por lo tanto, se desarrollarán nuevos modos de lectura, ya que se han desarrollado nuevos modos de lectura con la forma impresa. Pensemos aquí en toda la controversia alemana sobre la lectura intensiva y extensa, que marcó el paso de una situación en la que uno tiene, por ejemplo, sólo cinco o seis libros en casa y a los que constantemente regresa: un método de leer parecido al de la biblia o una forma de mirar un libro de medicina o cocina, porque necesitas encontrar recetas para resolver problemas, etc. Por otro lado, está la situación en la que consumimos libros, donde comenzamos a tener cientos de libros en casa: los leemos una vez, e incluso a veces los compramos y los guardamos en el estante, sin llegar nunca el momento de leerlos, porque no teníamos tiempo. ${ }^{12}$ Este tipo de transición de un modo de lectura a otro nos permite pensar que volverá a aparecer otro modo de lectura. Esto estará relacionado con lo que las máquinas podrán darnos para guiarnos.

A. L.: Este objetivo de estar lo más cerca posible de lo que se estaba en el corazón de los experimentos de los pioneros del acceso abierto y que permitió a los investigadores reclamar los medios de publicación también plantea preguntas de capacitación.

J.-C. G.: Sí, absolutamente. 
A. L.: Pero planteo esto más como un desafío que como un argumento contra el acceso abierto, por supuesto.

J.-C. G.: Ésta es una muy buena pregunta para agregar. Probablemente es en este contexto donde surge la cuestión de la plataforma. Para construir una plataforma óptima no es necesario imponerla "de arriba a abajo" a los investigadores, sino convertirla en una cuestión de gobernanza entre aquellos que probablemente reprocesen y mejoren esta herramienta y quienes la usen para comunicarse a través de ella. Es cierto que los experimentos de los pioneros permitieron repetir las ventajas de una cierta división del trabajo, sin embargo, todo el proceso de producción de conocimiento tiene una cierta unidad que debe resolverse mediante una metaconversación en relación con la conversación científica en sí y con respecto al diseño de la plataforma. Esta metaconversación forma precisamente el corazón del gobierno al que acabo de aludir.

A. L.: Ésta es una pregunta que debe abordarse porque algunos denuncian la "ilusión de la facilidad" del mundo digital, para ponerlo en palabras de Philippe Minard. ${ }^{13}$

J.-C. G.: Hablando de la ilusión de la facilidad digital, creo que debemos tener cuidado con lo que queremos decir. Está claro que en la década de 1990 los investigadores, al igual que yo, buscaban explorar lo que podíamos hacer solos en términos de la difusión de los resultados de la investigación. De esta primera ola de intentos de apropiación de la publicación en modo digital con Internet nació, por un lado, un conocimiento mucho mejor del mundo de la publicación científica y, por otro lado, una mejor comprensión de los oficios y habilidades necesarias para llevar a cabo esta difusión del conocimiento científico.

Sobre el primer punto, la economía política del mundo de las publicaciones científicas se ha vuelto mucho más clara, y esto ha motivado a muchos investigadores a involucrarse en el acceso abierto. Con respecto al segundo punto, los investigadores comprendieron rápidamente que muchas 
habilidades importantes no estaban necesaria y fácilmente del lado de los propios investigadores. Por otro lado, esta misma observación hizo posible ver rápidamente que, en las universidades, un nuevo "trabajador colectivo" (estoy utilizando aquí una vieja noción de la sociología del trabajo) podría construirse reorganizando las tareas de los bibliotecarios y prensas universitarias, y agregándoles habilidades que se encuentren en los departamentos de TI. Al mismo tiempo, esta conciencia hizo más fácil ver que las editoriales, lejos de constituir un todo unificado y homogéneo, en realidad obedecen a un conjunto de habilidades que podrían disociarse en sus servicios.

Este razonamiento conduce a un panorama muy diferente del que tiene como objetivo preservar a las editoriales en su papel de "mediador" indispensable y monolítico, para más bien constituir procesos de producción de publicaciones que, efectivamente, en tal y tal punto específico pueden requerir la provisión de un servicio que una sola empresa comercial puede proporcionarles. Esto incidentalmente corresponde a lo que sucede en los laboratorios: No fabrican todos sus instrumentos, también los compran, incluso si eso significa adaptarlos a su situación.

La realidad es que cuando se realizan publicaciones científicas internas, se descubre rápidamente que todas las habilidades y servicios necesarios están disponibles. Es suficiente ver cómo las bibliotecas se embarcan cada vez más en la publicación de monografías o revistas científicas. Y al igual que las editoriales comerciales, su problema radica más en las innovaciones necesarias para desbloquear todo su potencial para tratar documentos digitales que en la capacidad de hacer lo que hacen los comercios. Este último paso será el más difícil y el más emocionante, pero creo que la solución radicará en la posibilidad de que estas instituciones operen en una red de escalas al menos nacionales, sino continentales. En Europa, las experiencias con la Comisión Europea son bastante fascinantes con respecto 
a esto, pero no debe olvidarse que en América Latina, con SciELO, Redalyc y Latindex, se ha allanado el camino para su estudio.

Así que pensar que el mundo digital corresponde a los sueños de algunos de nosotros alrededor de 1990 es, de hecho, una ilusión de tranquilidad, pero para la desgracia de los soñadores que éramos en ese momento, hemos aterrizado en el continente digital y llevamos a cabo los primeros pasos en un territorio aún desconocido. Nuestras experiencias, si no nuestros fracasos, han contribuido a una mejor comprensión de la publicación digital académica. Las fallas y las experiencias también nos han enseñado que el mundo digital, sus herramientas, su potencial y los territorios que permite abrir son congruentes con la idea de que los investigadores y sus socios (bibliotecarios, prensas universitarias, informáticos, organizaciones y becarios de investigación) pueden unir fuerzas de una manera sin precedentes para permitir que el mundo de la investigación recupere el control sobre sus medios de comunicación. El destino de la gran conversación científica depende de ello.

\section{NOTAS}

${ }^{1}$ Guédon, Jean-Claude, Open Access: Toward the Internet of the Mind. Disponible en: http://www.budapestopenaccessinitiative.org/open-access-toward-the-internetof-the-mind. Este texto fue compuesto para el decimoquinto aniversario de la Declaración de Budapest.

${ }^{2}$ Gingras, Yves, L'impossible dialogue. Sciences et religions, París, PUF, 2016.

3 Guédon, Jean-Claude, In Oldenburg's Long Shadow Librarians, Research Scientists, Publishers, and The Control of Scientific Publishing, Washington, DC, Association of Research Libraries, 2001. Una traducción en francés está disponible en el siguiente enlace: https://hal.archives-ouvertes.fr/halshs-00395366/document.

${ }^{4}$ Se puede encontrar una presentación excelente del vínculo entre la plataforma y el "régimen de verdad" en el seminario de Olivier Ertzscheid, "Plates-formes et 
production de contenu", proporcionado de París a Montreal bajo el paraguas de la cátedra de investigación en escritura digital, ocupada por el excelente colega, Marcello Vitali-Rosati. Disponible en: https://www.youtube.com/watch?v $=$ DcIV3BymM2Q.

${ }^{5}$ Cfr. Stern, Niels; Guédon, Jean-Claude y Wiben, Thomas, "Crystals of Knowledge Production. An Intercontinental Conversation about Open Science and the Humanities", Nordic Perspectives en Open Science, 2015(1). Disponible en: http://dx.doi.org/10.7557/11.3619.

${ }^{6}$ Minard, Philippe, "Les revues à l'âge numérique : au péril de l'idéologie", Revue d'Histoire Moderne et Contemporaine, 2015/5, núm. 62-4 bis, pp. 8-21.

${ }^{7}$ Chartron, Ghislaine, "Open access et SHS: controverses", Revue Européenne des Sciences Sociales, 52-1, 2014, pp. 37-63.

${ }^{8}$ Cfr. Calafat, Guillaume y Monnet, Eric, "A la recherche de l'accès ouvert, Revues et nouveaux formats numériques", Revue d'Histoire Moderne et Contemporaine, 2015/5, núm. 62-4 bis, pp. 33-42.

${ }^{9}$ Cfr. Stern, Niels; Guédon, Jean-Claude y Wiben, Thomas, "Crystals of Knowledge Production. An Intercontinental Conversation about Open Science and the Humanities", Nordic Perspectives en Open Science, 2015(1). Disponible en: http://dx.doi.org/10.7557/11.3619.

${ }^{10}$ Chartier, Roger, 'Qu'est-ce qu'un livre ? Métaphores anciennes, concepts des lumières et réalités numériques", Le Français Aujourd'hui, 2012/3, núm. 178, pp. 11 26.

${ }^{11}$ Darnton, Robert, Apologie du livre, París, Gallimard, 2012.

12 Guglielmo, Cavallo y Chartier, Roger, Histoire de la lecture dans le monde occidental, París, Éditions du Seuil, 1997.

${ }^{13}$ Minard, Philippe, "Les revues à l'âge numérique: au péril de l'idéologie", Revue d'Histoire Moderne et Contemporaine, 2015/5, núm. 62-4 bis, pp. 8-21. 


\section{RESUMEN}

Publicamos aquí la transcripción de una entrevista a Jean-Claude Guédon realizada en Montreal el 8 de mayo de 2017. La entrevista se estructura en dos etapas. Comienza con un monólogo a través del cual Jean-Claude Guédon recorre, en cuatro etapas, la historia del desarrollo de las revistas científicas. Luego viene un intercambio de preguntas y respuestas sobre diversos temas, como el acceso abierto, la relación con la política o el desafío de la herencia de las formas mediáticas de las publicaciones científicas. A propósito de éstas, hemos realizado un seguimiento de la naturaleza oral del intercambio. Nos gustaría agradecer calurosamente a Jean-Claude Guédon por su disponibilidad y por el largo tiempo que nos dedicó para la entrevista.

\section{AUTORES}

Jean-Claude Guédon

Jean-Claude Guédon es profesor en la Universidad de Montreal desde 1973. Obtuvo su Doctorado en Historia de la Ciencia en la Universidad de WisconsinMadison en 1974, con una tesis sobre química en la Encyclopédie de Diderot et d'Alembert. Fue uno de los signatarios de la Declaración de Budapest en febrero de 2002, a menudo considerada como el origen del movimiento para el acceso abierto a las publicaciones científicas. Es experto en la Comisión Europea desde 2008 y actualmente preside un grupo de expertos sobre el futuro de las publicaciones científicas. También es "fideicomisario" del centro Nexa-Internet and Society del Politécnico de Turín.

\section{Alain Loute}

Alain Loute es doctor en Filosofía, es profesor en el Centro de Ética Médica, EA7446 "ETHICS: Ethics on Experiments, Transhumanism, Human Interactions, Care and 
Societ", en la Universidad Católica de Lille. Trabaja en el campo de la ética de la innovación tecnológica, la salud digital, la ética del cuidado y la hermenéutica. De manera particular, ha publicado La création sociale des normes. De la socioéconomie des conventions à la philosophie de l'action de Paul Ricoeur (Olms, 2008) y coeditado, con Marc Maesschalck, Nouvelle critique sociale, Europe-Amérique Latine, Aller-Retour (Polimetrica, 2011). Recientemente ha coeditado, con Louis Carré, Donner, reconnaitre, dominer. Trois modèles en philosophie sociale (Septentrion 2016). Fue investigador en el Fondo Nacional de Investigación Científica, así como facilitador de educación permanente en el Centro de Información y Educación Popular del Movimiento Obrero Cristiano. 